\title{
Semidefinite Relaxation Algorithm for Multisource Localization Using TDOA Measurements with Range Constraints
}

\author{
Changgui Jia, ${ }^{1,2}$ Jiexin Yin $\mathbb{D}^{1,2}$ Ding Wang $\mathbb{D}^{1,2}$ Yunlong Wang, ${ }^{1,2}$ and Li Zhang ${ }^{1,2}$ \\ ${ }^{1}$ National Digital Switching System Engineering and Technology Research Center, Zhengzhou 450002, China \\ ${ }^{2}$ Zhengzhou Information Science and Technology Institute, Zhengzhou, Henan 450002, China \\ Correspondence should be addressed to Jiexin Yin; Cindyin0807@163.com
}

Received 18 April 2018; Accepted 4 June 2018; Published 28 June 2018

Academic Editor: David Plets

Copyright (c) 2018 Changgui Jia et al. This is an open access article distributed under the Creative Commons Attribution License, which permits unrestricted use, distribution, and reproduction in any medium, provided the original work is properly cited.

\begin{abstract}
Multiple sources localization based on time difference of arrival (TDOA) measurements is investigated in this paper. Different from the traditional methods, a novel and practical multisource localization algorithm is proposed by adopting a priori information of relative distance among emitting sources. Since the maximum likelihood (ML) cost function for multisource estimation is highly nonconvex, the semidefinite relaxation (SDR) is utilized to reformulate the ML cost function. A robust estimator is obtained, which can be solved by semidefinite programming (SDP). Moreover, the constrained Cramér-Rao bound is also derived as a benchmark by considering the range constraints between sources. Simulation results verify the superior performance of the proposed algorithm over the traditional methods.
\end{abstract}

\section{Introduction}

Multisource localization is an essential task in radar, sonar, navigation, and other applications $[1,2]$. According to $[3$, 4], time difference of arrival (TDOA) measurements can be utilized to locate a source with high accuracy, which circumvents the synchronization problem in the time of arrival- (TOA-) based methods.

The positioning problem of using TDOA measurements is a nontrivial task due to its high nonlinearity and nonconvexity. In [3], the authors develop an iterative nonlinear leastsquares (NLS) algorithm based on Taylor-series expansion, but it requires sufficiently precise initial estimates for the global solution. Otherwise, it may suffer from the problem of local convergence. To alleviate this drawback, a twostep weighted least-squares (WLS) method is put forward to approximate the maximum likelihood (ML) function and a closed-form solution is also obtained [4]. However, inaccurate estimation of the covariance matrix of measurement errors used in [4] can result in performance degradation in practice. In general, the least-squares (LS) methods can achieve the Cramér-Rao bound (CRB) when the measurement errors are sufficiently small.
Since the convex optimization has been applied to solve localization problems, many researchers prove that this method is attractive to robustly achieve excellent estimate results and accuracy even at high noise levels [5-8]. This is mainly because of its preferable property of providing globally optimal solution without needing any initial estimates and efficient calculation with existing software package. Usually, there are two ways to apply the semidefinite relaxation (SDR) techniques. The authors in [5] start from reformulating the nonconvex ML problem to a convex optimization problem and then add a constraint based on a priori knowledge of the admissible source position. Another approach is presented in [6-8] where the robust LS cost function is firstly established and then relaxed to a semidefinite programming (SDP) problem. It is worth pointing out that these meaningful trials lay the foundations for our further exploration of using the SDR techniques. Despite the preceding progress, all of these works consider only single source.

Multisource localization is of great interest for its frequent emergence in practice. Here we address the problem in some common scenarios where emitting sources lie within a certain range or move in group, which can be referred to as group targets. The targets are typically sensors in the wireless sensor 
network or formation-flight aircraft and aircraft carrier fleet in the open space. Such patterns usually indicate that these group targets will stay within a certain relative distance with each other and keep the resemble velocity for most of the times. For example, aircraft in formation-flight usually keeps a distance around several meters to tens of meters between each companion in the formation [9], and it is also similar for group targets with unknown positions and velocities in sensor network.

To date, only several papers have considered the problem of localizing multiple sources [1, 2, 10-14]. One of the challenges for multisource localization is data association of multisensor and multisource. When the sensors have no prior knowledge of individual signal features of the sources, namely, the associations between the multiple signal measurements at each sensor and the corresponding sources are unknown, a target acquisition and localization algorithm (TALA) is developed in [10], which utilizes hybrid angle-ofarrival (AOA) and TOA measurements generated within a time window by an array of sensors to detect and localize an unknown number of targets. The authors in [11] apply TDOA measurements to localize multiple acoustic sources by treating the association problem within a multiple-hypothesis framework. Alternatively, using TOA measurements, [12, 13] proposes a three-stage algorithm to simultaneously estimate the source-measurement associations and the sources locations, respectively. There are also other cases when the problem of matching measurement data with the original emitting sources can be accomplished through techniques that make use of the disjointness of different sources [1], such as time, frequency, or signal transients [2]. Hence, the positioning parameters for multisource, e.g., TDOA, can be obtained separately. Based on the perfect matching, the multisource localization algorithms in $[1,14]$ exploit the fact that the TDOAs from different sources have the same sensor position displacements to jointly estimate the sources positions and improve the localization accuracy when the sensor positions are not known exactly. However, the prior information of relative distance among sources has not been considered in these related works.

In this paper, the number of sources to be located is assumed to be already known and the associations between the multiple signal measurements at each sensor and the corresponding sources have been accomplished. Unlike the traditional approaches mentioned above, this paper devises a novel method using SDR techniques to jointly locate the multiple sources by introducing the prior information constraints on relative distances between them. Practically, these relative distance information could be obtained through other observation methods. And it can improve the localization accuracy with such range constraints for group targets. The contributions of this paper are summarized as follows.

(1) We formulate the TDOA-based ML problem for multisource localization as an SDP problem which can be solved by the convex optimization tools. The tightness of our SDP is also enhanced by introducing the existing methods, such as utilizing Cauchy-Schwartz inequality.

(2) The prior information of relative distance among sources is introduced as convex constraint for group targets and its effectiveness of improving localization accuracy is verified by our simulations.

(3) The constrained CRB is derived in this paper as a benchmark to evaluate the proposed method by incorporating the prior range constraints.

The rest of the paper is organized as follows. In Section 2, the TDOA measurement model for multisource is given. Subsequently, the semidefinite relaxation (SDR) method for solving the localization problems is presented in Section 3 and complexity analysis of the proposed algorithm is given in Section 4. Section 5 derives the constrained CRB with inequality constraints. Simulation results are illustrated in Section 6. Finally, conclusions are drawn in Section 7.

In the sequel of this paper, boldface lowercase letters represent column vectors and boldface uppercase letters denote matrices. $\mathbf{a}^{o}$ is used to denote the noise-free value of $\mathbf{a} .(\bullet)^{\mathrm{T}}$ denotes transpose operator and $\widehat{\mathbf{a}}$ represents the estimation of a. blkdiag $\{\mathbf{A}, \mathbf{B}, \ldots, \mathbf{C}\}$ constructs block diagonal matrix from the matrices $\mathbf{A}, \mathbf{B}$, and $\mathbf{C}$. $\operatorname{tr}(\mathbf{A})$ means the trace of $\mathbf{A}$, and $\mathbf{A} \succeq \mathbf{B}$ means that $\mathbf{A}-\mathbf{B}$ is positive semidefinite.

\section{Problem Formulation}

Consider that there are $M$ sensors deployed at the known positions as $\mathbf{s}_{i}(i=1,2, \ldots, M)$ to intercept signals emitted by $N$ sources at unknown positions denoted by $\mathbf{u}_{j}(j=$ $1,2, \ldots, N)$ in the three-dimensional (3D) scenario. Usually we have $M \geq 4$ and $N \geq 2$. The unknown parameter vector to be determined is denoted by $\mathbf{u}=\left[\mathbf{u}_{1}^{\mathrm{T}}, \mathbf{u}_{2}^{\mathrm{T}}, \ldots, \mathbf{u}_{N}^{\mathrm{T}}\right]^{\mathrm{T}}$. Note that the vector $\mathbf{u}$ is a necessity to construct our SDP in the following sections.

Without loss of generality, the first sensor is chosen as the reference sensor and line-of-sight propagation condition is considered. The TDOA measurement between a sensor pair $i$ and 1 from source $j$ is given by

$$
\begin{aligned}
\tau_{i 1, j}=\frac{1}{c}\left\|\mathbf{u}_{j}-\mathbf{s}_{i}\right\|-\frac{1}{c}\left\|\mathbf{u}_{j}-\mathbf{s}_{1}\right\|+\Delta \tau_{i 1, j}, & \\
& i=2,3, \ldots, M, j=1,2, \ldots, N,
\end{aligned}
$$

where $c$ is the signal propagation speed. $\Delta \tau_{i 1, j}$ denotes the TDOA measurement noise that is assumed to be zero-mean Gaussian distributed. $\left\|\mathbf{u}_{j}-\mathbf{s}_{i}\right\|$ represents the distance between $j^{\text {th }}$ source and $i^{\text {th }}$ sensor and $\|\bullet\|$ is Euclid norm. Multiplying $c$ and TDOA measurement in (1), the range difference of arrival (RDOA) is obtained by

$$
\begin{aligned}
r_{i 1, j}=\left\|\mathbf{u}_{j}-\mathbf{s}_{i}\right\|-\left\|\mathbf{u}_{j}-\mathbf{s}_{1}\right\|+\Delta r_{i 1, j}, & \\
& i=2,3, \ldots, M, j=1,2, \ldots, N,
\end{aligned}
$$

where $\Delta r_{i 1, j}=c \Delta \tau_{i 1, j}$. Since there are $M$ sensors, the collection of RDOA measurements for source $j$ is $\mathbf{r}_{j}=\left[r_{21, j}, r_{31, j}, \ldots\right.$, $\left.r_{M 1, j}\right]^{\mathrm{T}}=\mathbf{r}_{j}^{o}+\Delta \mathbf{r}_{j}$, where $\Delta \mathbf{r}_{j}=\left[\Delta r_{21, j}, \Delta r_{31, j}, \ldots, \Delta r_{M 1, j}\right]^{\mathrm{T}}$ is the noise vector and

$$
\begin{aligned}
\mathbf{r}_{j}^{o} & =\left[\left\|\mathbf{u}_{j}-\mathbf{s}_{2}\right\|-\left\|\mathbf{u}_{j}-\mathbf{s}_{1}\right\|,\left\|\mathbf{u}_{j}-\mathbf{s}_{3}\right\|\right. \\
& \left.-\left\|\mathbf{u}_{j}-\mathbf{s}_{1}\right\|, \ldots,\left\|\mathbf{u}_{j}-\mathbf{s}_{M}\right\|-\left\|\mathbf{u}_{j}-\mathbf{s}_{1}\right\|\right]^{\mathrm{T}} .
\end{aligned}
$$


Then collect all measurements of $N$ sources, and the $N(M-1) \times 1$ measurement vector is denoted by

$$
\mathbf{r}=\left[\mathbf{r}_{1}^{\mathrm{T}}, \mathbf{r}_{2}^{\mathrm{T}}, \ldots, \mathbf{r}_{N}^{\mathrm{T}}\right]^{\mathrm{T}}=\mathbf{r}^{o}+\Delta \mathbf{r}
$$

where $\Delta \mathbf{r}=\left[\Delta \mathbf{r}_{1}^{\mathrm{T}}, \Delta \mathbf{r}_{2}^{\mathrm{T}}, \ldots, \Delta \mathbf{r}_{N}^{\mathrm{T}}\right]^{\mathrm{T}}$ and $\mathbf{r}^{o}=\left[\mathbf{r}_{1}^{o \mathrm{~T}}, \mathbf{r}_{2}^{o \mathrm{~T}}, \ldots\right.$, $\left.\mathbf{r}_{N}^{o \mathrm{~T}}\right]^{\mathrm{T}}$. Furthermore, $\Delta \mathbf{r}$ is assumed to be a zero-mean Gaussian random vector with covariance matrix $\mathbf{Q}_{\Delta}=$ $\mathbf{E}\left[\Delta \mathbf{r} \Delta \mathbf{r}^{\mathrm{T}}\right]$. Using similar formation method in [7], $\mathbf{r}^{o}$ can be reformulated as

$$
\mathbf{r}^{o}=\mathbf{G d}
$$

where the $N M \times 1$ vector:

$$
\begin{gathered}
\mathbf{d}=\left[\left\|\mathbf{u}_{1}-\mathbf{s}_{1}\right\|, \ldots,\left\|\mathbf{u}_{1}-\mathbf{s}_{M}\right\|, \ldots,\left\|\mathbf{u}_{N}-\mathbf{s}_{1}\right\|, \ldots,\right. \\
\left.\left\|\mathbf{u}_{N}-\mathbf{s}_{M}\right\|\right]^{\mathrm{T}},
\end{gathered}
$$

and the $N(M-1) \times N M$ matrix $\mathbf{G}=\operatorname{blkdiag}\left\{\left[\begin{array}{ll}-\mathbf{1}_{M-1} & \mathbf{I}_{M-1}\end{array}\right]\right.$, $\left.\ldots,\left[\begin{array}{ll}-\mathbf{1}_{M-1} & \mathbf{I}_{M-1}\end{array}\right]\right\} . \mathbf{1}_{M-1}$ and $\mathbf{I}_{M-1}$ denote the $(M-1) \times 1$ all one column vector and the $(M-1) \times(M-1)$ identity matrix, respectively. We can further rewrite (4) as

$$
\mathbf{r}=\mathbf{G} \mathbf{d}+\Delta \mathbf{r}
$$

Then the problem of interest is to estimate the unknown vector $\mathbf{u}$ given the RDOA measurements $\mathbf{r}$ and the prior information of relative distance among sources. Here the prior range constraints for multiple sources that accompany each other in group are introduced. As interpreted before, group targets usually maintain a certain formation where the relative distances among each other can be bounded with a certain value, and the range constraints are given by

$$
\left\|\mathbf{u}_{j}-\mathbf{u}_{m}\right\| \leq \delta, \quad j, m=1,2, \ldots, N
$$

where $\delta$ is the upper range bound between two targets. Similar idea can also be found in [15], but it just considers the potential communication range of an anchor to confine an area that a source must lie within. Here we practically consider a rough upper bound for all potential relative distances among sources since they are not equal or may change slightly in real situations. Besides, the loose upper range bound can be tightened through more precise observations. Specifically, the selection of the upper range bound parameter $\delta$ in practice can be accomplished by the following ways:

(1) If the group targets are in the open space, we can obtain a rough target position by some preestimation methods $[3,5]$, separately. Then the relative distances between targets can be computed using the preestimated target positions. Next, based on the relative distances obtained through the preestimation process, we can select a large, but appropriate, $\delta$ to establish the range constraints.

(2) For a group of targets that exist in a formation group [9], the relative distances between the targets can be obtained according to the corresponding formation rules and types, and then the value of $\delta$ can be selected with proper consideration.

\section{Semidefinite Relaxation Method for Multisource Localization}

In this section, the SDR techniques are employed to approximate the ML problem for multiple sources localization and incorporate the range inequalities as convex constraints. According to (7), the ML estimation of $\mathbf{u}$ can be formulated as

$$
\widehat{\mathbf{u}}=\arg \min _{\mathbf{u}_{1}, \ldots, \mathbf{u}_{N} \in \mathbb{R}^{3}}(\mathbf{r}-\mathbf{G d})^{\mathrm{T}} \mathbf{Q}_{\Delta}^{-1}(\mathbf{r}-\mathbf{G d}) .
$$

Then, (9) can be rewritten as a constrained quadratic program as follows:

$$
\begin{aligned}
\min _{\mathbf{u}_{1}, \ldots, \mathbf{u}_{N}, \mathbf{d}} & (\mathbf{r}-\mathbf{G d})^{\mathrm{T}} \mathbf{Q}_{\Delta}^{-1}(\mathbf{r}-\mathbf{G d}), \\
\text { s.t. } \quad & {[\mathbf{d}]_{M(j-1)+i}^{2}=\left\|\mathbf{u}_{j}-\mathbf{s}_{i}\right\|^{2}, } \\
& {[\mathbf{d}]_{M(j-1)+i} \geq 0, } \\
& i=1,2, \ldots, M, j=1,2, \ldots, N .
\end{aligned}
$$

It is proved in [16] that (10c) can be neglected since they are always satisfied for any globally optimal solution determined by (10a) and (10b). Additionally, the objective function in (10a) is convex, and the quadratic equality constraints in (10b) are nonconvex. To transform the problem to standard convex optimization problem defined in [17], problem (10a) and (10b) can be equivalently reexpressed by decomposing the Euclid norms as

$$
\begin{aligned}
& \underset{\mathbf{u}_{1}, \ldots, \mathbf{u}_{N}, \mathbf{d}}{\min } {\left[\begin{array}{l}
\mathbf{d} \\
1
\end{array}\right]^{\mathrm{T}}\left[\begin{array}{cc}
\mathbf{G}^{\mathrm{T}} \mathbf{Q}_{\Delta}^{-1} \mathbf{G} & -\mathbf{G}^{\mathrm{T}} \mathbf{Q}_{\Delta}^{-1} \mathbf{r} \\
-\mathbf{r}^{\mathrm{T}} \mathbf{Q}_{\Delta}^{-1} \mathbf{G} & \mathbf{r}^{\mathrm{T}} \mathbf{Q}_{\Delta}^{-1} \mathbf{r}
\end{array}\right]\left[\begin{array}{l}
\mathbf{d} \\
1
\end{array}\right], } \\
& \text { s.t. } \quad[\mathbf{d}]_{M(j-1)+i}^{2}=\left[\begin{array}{c}
\mathbf{u}_{j} \\
1
\end{array}\right]^{\mathrm{T}}\left[\begin{array}{cc}
\mathbf{I} & -\mathbf{s}_{i} \\
-\mathbf{s}_{i}^{\mathrm{T}} & \mathbf{s}_{i}^{\mathrm{T}} \mathbf{s}_{i}
\end{array}\right]\left[\begin{array}{c}
\mathbf{u}_{j} \\
1
\end{array}\right], \\
& i=1,2, \ldots, M, j=1,2, \ldots, N .
\end{aligned}
$$

By applying the basic property of $\mathbf{x}^{\mathrm{T}} \mathbf{A} \mathbf{x}=\operatorname{tr}\left\{\mathbf{x} \mathbf{x}^{\mathrm{T}} \mathbf{A}\right\}$, (11a) and (11b) can be reformulated as

$$
\begin{array}{ll}
\min _{\mathbf{u}, \mathbf{d}, \mathbf{U}, \mathbf{D}} & \operatorname{tr}\left\{\left[\begin{array}{ll}
\mathbf{D} & \mathbf{d} \\
\mathbf{d}^{\mathrm{T}} & 1
\end{array}\right] \mathbf{F}\right\}, \\
\text { s.t. } & {[\mathbf{D}]_{M(j-1)+i, M(j-1)+i}} \\
& =\operatorname{tr}\left\{\left[\begin{array}{ll}
\mathbf{J}_{j} \mathbf{U} \mathbf{J}_{j}^{\mathrm{T}} & \mathbf{J}_{j} \mathbf{u} \\
\left(\mathbf{J}_{j} \mathbf{u}\right)^{\mathrm{T}} & 1
\end{array}\right]\left[\begin{array}{cc}
\mathbf{I} & -\mathbf{s}_{i} \\
-\mathbf{s}_{i}^{\mathrm{T}} & \mathbf{s}_{i}^{\mathrm{T}} \mathbf{s}_{i}
\end{array}\right]\right\}, \\
\mathbf{D} & =\mathbf{d d}^{\mathrm{T}}, \\
\mathbf{U} & =\mathbf{u} \mathbf{u}^{\mathrm{T}}, \\
& i=1,2, \ldots, M, j=1,2, \ldots, N,
\end{array}
$$

where $\mathbf{F}=\left[\begin{array}{ccc}\mathbf{G}^{\mathrm{T}} \mathbf{Q}_{\Delta}^{-1} \mathbf{G} & -\mathbf{G}^{\mathrm{T}} \mathbf{Q}_{\Delta}^{-1} \mathbf{r} \\ -\mathbf{r}^{\mathrm{T}} \mathbf{Q}_{\Delta}^{-1} \mathbf{G} & \mathbf{r}^{\mathrm{T}} \mathbf{Q}_{\Delta}^{-1} \mathbf{r}\end{array}\right], \mathbf{J}_{j}=\left[\begin{array}{lll}\mathbf{0}_{3 \times 3(j-1)} & \mathbf{I}_{3} & \mathbf{0}_{3 \times 3(N-j)}\end{array}\right]$ is the $3 \times 3 N$ matrix, and it is easy to check that $\mathbf{u}_{j}=\mathbf{J}_{j} \mathbf{u}$. 
Using the SDR principle, we relax the constraints $\mathbf{D}=$ $\mathbf{d d}^{\mathrm{T}}$ as $\mathbf{D} \succeq \mathbf{d d}^{\mathrm{T}}$ and $\mathbf{U}=\mathbf{u u}^{\mathrm{T}}$ as $\mathbf{U} \succeq \mathbf{u u}^{\mathrm{T}}$, respectively. By utilizing the basic property of Schur-complements described in [17], these relaxed constraints can be equivalently rewritten as

$$
\begin{aligned}
& {\left[\begin{array}{ll}
\mathbf{D} & \mathbf{d} \\
\mathbf{d}^{\mathrm{T}} & 1
\end{array}\right] \geq 0,} \\
& {\left[\begin{array}{ll}
\mathbf{U} & \mathbf{u} \\
\mathbf{u}^{\mathrm{T}} & 1
\end{array}\right] \geq 0,}
\end{aligned}
$$

where the matrices are of rank 1 and symmetric positive semidefinite (PSD). Note that the PSD constraints in (13) are convex [17]. Then we obtain a convex optimization problem which can be seen as an approximation of the ML problem described in (9).

It is necessary to point out that, as shown in [5], such relaxations are not tight enough to yield highly precise solutions. In order to remedy the SDR formulation to enhance the tightness and also make use of the prior information of relative distance among sources, two types of additional constraints for $\mathbf{D}$ and $\mathbf{U}$ are devised subsequently.

We consider using the relationship between elements of $\mathbf{D}$ and $\mathbf{d \mathbf { d } ^ { T }}$ to generate the constraints for unknown parameters to be determined as the first type. Since there are $M$ receivers and by Cauchy-Schwartz inequality, for $j^{\text {th }}$ source we have

$$
\begin{aligned}
{[\mathbf{D}]_{M(j-1)+i, M(j-1)+n} } & =[\mathbf{d}]_{M(j-1)+i}[\mathbf{d}]_{M(j-1)+n} \\
& =\left\|\mathbf{u}_{j}-\mathbf{s}_{i}\right\|\left\|\mathbf{u}_{j}-\mathbf{s}_{n}\right\| \\
& \geq\left|\left(\mathbf{u}_{j}-\mathbf{s}_{i}\right)^{\mathrm{T}}\left(\mathbf{u}_{j}-\mathbf{s}_{n}\right)\right|,
\end{aligned}
$$

where

$$
\begin{gathered}
\left(\mathbf{u}_{j}-\mathbf{s}_{i}\right)^{\mathrm{T}}\left(\mathbf{u}_{j}-\mathbf{s}_{n}\right)=\left[\begin{array}{c}
\mathbf{u}_{j} \\
1
\end{array}\right]^{\mathrm{T}}\left[\begin{array}{cc}
\mathbf{I} & -\mathbf{s}_{n} \\
-\mathbf{s}_{i}^{\mathrm{T}} & \mathbf{s}_{i}^{\mathrm{T}} \mathbf{s}_{n}
\end{array}\right]\left[\begin{array}{c}
\mathbf{u}_{j} \\
1
\end{array}\right] \\
=\operatorname{tr}\left\{\left[\begin{array}{cc}
\mathbf{J}_{j} \mathbf{U} \mathbf{J}_{j}^{\mathrm{T}} & \mathbf{J}_{j} \mathbf{u} \\
\left(\mathbf{J}_{j} \mathbf{u}\right)^{\mathrm{T}} & 1
\end{array}\right]\left[\begin{array}{cc}
\mathbf{I} & -\mathbf{s}_{n} \\
-\mathbf{s}_{i}^{\mathrm{T}} & \mathbf{s}_{i}^{\mathrm{T}} \mathbf{s}_{n}
\end{array}\right]\right\} .
\end{gathered}
$$

Therefore, it is straightforward to obtain the following inequalities:

$$
\begin{aligned}
& {[\mathbf{D}]_{M(j-1)+i, M(j-1)+n}} \\
& \geq\left|\operatorname{tr}\left\{\left[\begin{array}{cc}
\mathbf{J}_{j} \mathbf{U J}_{j}^{\mathrm{T}} & \mathbf{J}_{j} \mathbf{u} \\
\left(\mathbf{J}_{j} \mathbf{u}\right)^{\mathrm{T}} & 1
\end{array}\right]\left[\begin{array}{cc}
\mathbf{I} & -\mathbf{s}_{n} \\
-\mathbf{s}_{i}^{\mathrm{T}} & \mathbf{s}_{i}^{\mathrm{T}} \mathbf{s}_{n}
\end{array}\right]\right\}\right|, \\
& \quad i, n=1,2, \ldots, M, n>i, j=1,2, \ldots, N .
\end{aligned}
$$

It is necessary to point out that the inequalities above impose constraints on the product of ranges for each source with different sensor pairs. That is, the correspondent elements that lie in the upper triangular matrix around the principle diagonal in $\mathbf{D}$ are restrained. The effectiveness of these inequality constraints will be verified through our simulations.
Then the prior information of relative distance in (8) for group targets can be utilized to form the second type of constraints. Using the above relations, (8) can be represented as

$$
\left\|\mathbf{J}_{j} \mathbf{u}-\mathbf{J}_{m} \mathbf{u}\right\| \leq \delta, \quad j, m=1,2, \ldots, N, j>m .
$$

Squaring both sides of (17) yields

$$
\left\|\mathbf{J}_{j} \mathbf{u}-\mathbf{J}_{m} \mathbf{u}\right\|^{2} \leq \delta^{2},
$$

which is equivalent to

$$
\mathbf{u}^{\mathrm{T}}\left(\mathbf{J}_{j}-\mathbf{J}_{m}\right)^{\mathrm{T}}\left(\mathbf{J}_{j}-\mathbf{J}_{m}\right) \mathbf{u} \leq \delta^{2} .
$$

Note that the quadratic inequality constraint in (19) is convex. By applying $\mathbf{x}^{\mathrm{T}} \mathbf{A} \mathbf{x}=\operatorname{tr}\left\{\mathbf{x} \mathbf{x}^{\mathrm{T}} \mathbf{A}\right\}$ to (19), we have

$$
\operatorname{tr}\left\{\mathbf{u} \mathbf{u}^{\mathrm{T}}\left(\mathbf{J}_{j}-\mathbf{J}_{m}\right)^{\mathrm{T}}\left(\mathbf{J}_{j}-\mathbf{J}_{m}\right)\right\} \leq \delta^{2} .
$$

Substituting $\mathbf{U}=\mathbf{u u}^{\mathrm{T}}$ into (20) obtains

$$
\operatorname{tr}\left\{\mathbf{U J}_{j m}\right\} \leq \delta^{2} \quad j, m=1,2, \ldots, N, j>m,
$$

where $\mathbf{J}_{j m}=\left(\mathbf{J}_{j}-\mathbf{J}_{m}\right)^{\mathrm{T}}\left(\mathbf{J}_{j}-\mathbf{J}_{m}\right)$. In summary, adding the constraints of (13), (16), and (21) to (12a), (12b), and (12c), the proposed SDP is obtained as follows:

$$
\begin{aligned}
& \min _{\mathbf{u}, \mathbf{d}, \mathbf{U}, \mathbf{D}} \operatorname{tr}\left\{\left[\begin{array}{ll}
\mathbf{D} & \mathbf{d} \\
\mathbf{d}^{\mathrm{T}} & 1
\end{array}\right] \mathbf{F}\right\}, \\
& \text { s.t. }[\mathbf{D}]_{M(j-1)+i, M(j-1)+i} \\
& =\operatorname{tr}\left\{\left[\begin{array}{cc}
\mathbf{J}_{j} \mathbf{U J}_{j}^{\mathrm{T}} & \mathbf{J}_{j} \mathbf{u} \\
\left(\mathbf{J}_{j} \mathbf{u}\right)^{\mathrm{T}} & 1
\end{array}\right]\left[\begin{array}{cc}
\mathbf{I} & -\mathbf{s}_{i} \\
-\mathbf{s}_{i}^{\mathrm{T}} & \mathbf{s}_{i}^{\mathrm{T}} \mathbf{s}_{i}
\end{array}\right]\right\}, \\
& \operatorname{tr}\left\{\mathbf{U J}_{j m}\right\} \leq \delta^{2} \quad j, m=1,2, \ldots, N, j>m, \\
& {[\mathbf{D}]_{M(j-1)+i, M(j-1)+n}} \\
& \geq\left|\operatorname{tr}\left\{\left[\begin{array}{cc}
\mathbf{J}_{j} \mathbf{U} \mathbf{J}_{j}^{\mathrm{T}} & \mathbf{J}_{j} \mathbf{u} \\
\left(\mathbf{J}_{j} \mathbf{u}\right)^{\mathrm{T}} & 1
\end{array}\right]\left[\begin{array}{cc}
\mathbf{I} & -\mathbf{s}_{n} \\
-\mathbf{s}_{i}^{\mathrm{T}} & \mathbf{s}_{i}^{\mathrm{T}} \mathbf{s}_{n}
\end{array}\right]\right\}\right| \\
& i, n=1,2, \ldots, M, n>i, j=1,2, \ldots, N, \\
& {\left[\begin{array}{ll}
\mathbf{D} & \mathbf{d} \\
\mathbf{d}^{\mathrm{T}} & 1
\end{array}\right] \geq 0 \text {, }} \\
& {\left[\begin{array}{cc}
\mathbf{U} & \mathbf{u} \\
\mathbf{u}^{\mathrm{T}} & 1
\end{array}\right] \succeq 0 \text {. }}
\end{aligned}
$$

This canonical convex optimization problem can be solved by CVX Toolbox in Matlab. By solving the SDP in (22), the estimation of $\mathbf{u}$ is finally obtained.

Remark 1. The covariance matrix $\mathbf{Q}_{\Delta}$ in $\mathbf{F}$ can be estimated by taking the covariance of the TDOA measurements obtained 
from multiple observations in practice. The corresponding estimation methods are described in detail in $[18,19]$. For clarity, the estimate of the element of $\mathbf{Q}_{\Delta}$ is given by

$$
\begin{aligned}
& {\left[\mathbf{Q}_{\Delta}\right]_{j \cdot(i-1), m \cdot(n-1)}} \\
& =\frac{1}{K-1} \cdot \sum_{k=1}^{K}\left(r_{i 1, j}[k]-\bar{r}_{i 1, j}\right)\left(r_{n 1, m}[k]-\bar{r}_{n 1, m}\right), \\
& \quad i, n=2,3, \ldots, M ; j, m=1,2, \ldots, N
\end{aligned}
$$

where

$$
\begin{gathered}
\bar{r}_{i 1, j}=\frac{1}{K} \cdot \sum_{k=1}^{K} r_{i 1, j}[k], \\
\bar{r}_{n 1, m}=\frac{1}{K} \cdot \sum_{k=1}^{K} r_{n 1, m}[k],
\end{gathered}
$$

$K$ is the number of observations, and $r_{i 1, j}[k]$ is the TDOA measurement of the $k^{\text {th }}$ observation.

Remark 2. Theoretically, in terms of the effect of the range constraints introduced for group targets, the constraints may have little effect to improve the estimation performance for group targets when the measurement noise is small. This is mainly because, at small measurement noise level, the position estimation results without constraints may just slightly deviate from the true target positions; thus they will probably fall into the feasible domain confined by the range constraints and meet the constraints. However, when the measurement noise grows larger, the position estimation results without constraints might deviate from the real target positions by a large amount with a high probability, which are likely to exceed the feasible domain. In this case, the range constraints will definitely confine and reduce the deviations and significantly decrease the estimation errors. Therefore, the improvement of the estimation performance can be more significant by utilizing the range constraints at large measurement noise level. This will be illustrated by the simulation results in Section 6.

\section{Complexity Analysis}

Computational complexity is analyzed in this section. Here, we apply the result of $[6,20]$ to analyze the computational complexity of the proposed multisource localization algorithm, denoted as "SDP-CL", in the $m$-dimensional scenario. The localization algorithm for single target using similar SDR method in [7] except for the range constraints in (21), denoted as "SDP-SL", and the classic two-step weighted leastsquare (TS-WLS) method [4] are also discussed. In Table 1, algorithm complexities in terms of the number of iterations and operations needed in each iteration are examined. From the table, it can be seen that the complexity grows with the number of targets to be estimated and our SDP algorithm is computationally higher than the classic TS-WLS. Thus it needs further refining [6]. It is necessary to point out that,
TABLE 1: Complexity comparison of different algorithms.

\begin{tabular}{lcc}
\hline Algorithm & Iteration Number & Operation per Iteration \\
\hline TS-WLS & 1 & $\mathrm{O}((2 m+1) M)$ \\
SDP-SL & $\mathrm{O}\left((2 m)^{1 / 2}\right)$ & $\mathrm{O}\left((m M)^{2}\right)$ \\
SDP-CL & $\mathrm{O}\left((2 N m)^{1 / 2}\right)$ & $\mathrm{O}\left((m N M)^{2}\right)$ \\
\hline
\end{tabular}

unlike single target positioning algorithm, which locates the targets in a decoupled manner, our approach is to localize multiple targets jointly. Although the complexity of the proposed algorithm increases, the corresponding localization performance can be promoted. This is verified by the simulation results in Section 6.

\section{The Constrained CRB}

The CRB is usually seen as a benchmark against which the statistical efficiency of any unbiased estimators can be compared. The CRBs for single source and multisource localization using TDOA measurements have been investigated in [5] and [1], respectively, but they do not consider the situation where inequality constraints are employed. Inspired by the work of Gorman et al. [21], we try to incorporate the range constraint inequalities and derive a constrained CRB (CCRB).

The probability density function (PDF) of jointly Gaussian distributed measurement noise $\Delta \mathbf{r}$ is subsequently given by

$$
p(\mathbf{r} \mid \mathbf{u})=\frac{\exp \left\{-(1 / 2)(\mathbf{r}-\mathbf{G} \mathbf{d})^{\mathrm{T}} \mathbf{Q}_{\Delta}^{-1}(\mathbf{r}-\mathbf{G} \mathbf{d})\right\}}{\sqrt{(2 \pi)^{N(M-1)} \operatorname{det}\left(\mathbf{Q}_{\Delta}\right)}} .
$$

The corresponding Fisher information matrix (FIM) is obtained as

$$
\begin{aligned}
\mathbf{F I M} & =-\mathbf{E}\left[\frac{\partial^{2} \ln (p(\mathbf{r} \mid \mathbf{u}))}{\partial \mathbf{u} \partial \mathbf{u}^{\mathrm{T}}}\right] \\
& =\left[\mathbf{G} \frac{\partial \mathbf{d}}{\partial \mathbf{u}^{\mathrm{T}}}\right]^{\mathrm{T}} \mathbf{Q}_{\Delta}^{-1}\left[\mathbf{G} \frac{\partial \mathbf{d}}{\partial \mathbf{u}^{\mathrm{T}}}\right],
\end{aligned}
$$

where $\partial \mathbf{d} / \partial \mathbf{u}^{\mathrm{T}}$ is the $N M \times 3 N$ Jacobian matrix defined as

$$
\frac{\partial \mathbf{d}}{\partial \mathbf{u}^{\mathrm{T}}}=\left[\begin{array}{cccc}
\frac{\left(\mathbf{u}_{1}-\mathbf{s}_{1}\right)^{\mathrm{T}}}{\left\|\mathbf{u}_{1}-\mathbf{s}_{1}\right\|} & \mathbf{0}_{1 \times 3} & \cdots & \mathbf{0}_{1 \times 3} \\
\vdots & \vdots & \ddots & \vdots \\
\frac{\left(\mathbf{u}_{1}-\mathbf{s}_{M}\right)^{\mathrm{T}}}{\left\|\mathbf{u}_{1}-\mathbf{s}_{M}\right\|} & \mathbf{0}_{1 \times 3} & \cdots & \mathbf{0}_{1 \times 3} \\
\vdots & \vdots & \ddots & \vdots \\
\mathbf{0}_{1 \times 3} & \mathbf{0}_{1 \times 3} & \cdots & \frac{\left(\mathbf{u}_{N}-\mathbf{s}_{1}\right)^{\mathrm{T}}}{\left\|\mathbf{u}_{N}-\mathbf{s}_{1}\right\|} \\
\vdots & \vdots & \ddots & \vdots \\
\mathbf{0}_{1 \times 3} & \mathbf{0}_{1 \times 3} & \cdots & \frac{\left(\mathbf{u}_{N}-\mathbf{s}_{M}\right)^{\mathrm{T}}}{\left\|\mathbf{u}_{N}-\mathbf{s}_{M}\right\|}
\end{array}\right]
$$


TABle 2: Positions of sensors (m).

\begin{tabular}{lcccccc}
\hline Sensor no. & 1 & 2 & 3 & 4 & 5 & 5 \\
\hline$x$ & 300 & 400 & 300 & 350 & -100 & 200 \\
$y$ & 100 & 150 & 500 & 200 & -100 & -300 \\
$z$ & 150 & 100 & 200 & 100 & -100 & -200 \\
\hline
\end{tabular}

The inequalities in (8) can be written in a functional inequality constraint of the form

$$
\begin{aligned}
& \mathbf{b}(\mathbf{u}) \\
& =\left[b_{1,2}(\mathbf{u}), \cdots, b_{1, N}(\mathbf{u}), \cdots, b_{i, j}(\mathbf{u}), \cdots, b_{N-1, N}(\mathbf{u})\right]^{\mathrm{T}} \\
& \leq \mathbf{0}_{N(N-1) / 2 \times 1}, \\
& b_{i, j}(\mathbf{u})=\left\|\mathbf{u}_{i}-\mathbf{u}_{j}\right\|-\delta, \quad i=1,2, \ldots, N-1 j=2,3, \ldots, N .
\end{aligned}
$$

The constrained parameter space $\Theta_{C}$ is defined by $\mathbf{b}(\mathbf{u})$, and it is composed of the equality constraint $\mathbf{b}_{\mathrm{eq}}(\mathbf{u})=\mathbf{0}$ and pure inequality constraint $\mathbf{b}_{\text {ineq }}(\mathbf{u})<\mathbf{0}$. When $\mathbf{u}$ is a regular point that lies in the interior of $\Theta_{C}$, namely, $\mathbf{u}$ belongs to the set $\left\{\mathbf{u}: \mathbf{b}_{\text {ineq }}(\mathbf{u})<\mathbf{0}\right\}$ where the equality constraint is inactive, it is demonstrated in [21] that the constrained CRB is identical to the unconstrained CRB. However, due to the existence of measurement errors, some points of the estimated parameter $\widehat{\mathbf{u}}$ are not regular. Hence they will not lie in the interior of $\Theta_{C}$. Consequently, the equality constraints $\mathbf{b}_{\mathrm{eq}}(\mathbf{u})=\mathbf{0}$ will take effect under such condition, thus leading to the bound reduction. For this case, the classical $\mathrm{CRB}$ is invalid due to the active equality constraints.

It is proved in [21] that, for any unbiased estimator $\mathbf{u}$ confined by inequality constraints (28), the estimator error covariance matrix satisfies the matrix inequality

$$
\Sigma_{\mathbf{u}} \geq \mathbf{B}_{\mathbf{u}}
$$

where

$$
\mathbf{B}_{\mathbf{u}}=\left[\nabla \mathbf{m}_{\mathbf{u}}\right] \mathbf{Q}_{\mathbf{u}}(\mathbf{F I M})^{-1}\left[\nabla \mathbf{m}_{\mathbf{u}}\right]^{\mathrm{T}}
$$

where $\nabla \mathbf{m}_{\mathbf{u}}$ is the $3 N \times 3 N$ identity matrix for unbiased estimator [21], and $\mathbf{Q}_{\mathbf{u}}$ is the $3 N \times 3 N$ matrix which is defined as follows:

$$
\begin{aligned}
\mathbf{Q}_{\mathbf{u}} & =\mathbf{I}-(\mathbf{F I M})^{-1}[\nabla \mathbf{b}(\mathbf{u})]^{\mathrm{T}}\left\{[\nabla \mathbf{b}(\mathbf{u})](\mathbf{F I M})^{-1}[\nabla \mathbf{b}(\mathbf{u})]^{\mathrm{T}}\right\}^{+}[\nabla \mathbf{b}(\mathbf{u})], \\
\nabla \mathbf{b}(\mathbf{u}) & =\frac{\partial \mathbf{b}(\mathbf{u})}{\partial \mathbf{u}^{\mathrm{T}}}=\left[\left(\frac{\partial b_{1,2}(\mathbf{u})}{\partial \mathbf{u}^{\mathrm{T}}}\right)^{\mathrm{T}} \ldots\left(\frac{\partial b_{1, N}(\mathbf{u})}{\partial \mathbf{u}^{\mathrm{T}}}\right)^{\mathrm{T}} \cdots\left(\frac{\partial b_{i, j}(\mathbf{u})}{\partial \mathbf{u}^{\mathrm{T}}}\right)^{\mathrm{T}} \ldots\left(\frac{\partial b_{N-1, N}(\mathbf{u})}{\partial \mathbf{u}^{\mathrm{T}}}\right)^{\mathrm{T}}\right]^{\mathrm{T}}, \\
\frac{\partial b_{i, j}(\mathbf{u})}{\partial \mathbf{u}^{\mathrm{T}}} & =\left[\mathbf{0}_{1 \times 3(i-1)} \frac{\left(\mathbf{u}_{i}-\mathbf{u}_{j}\right)^{\mathrm{T}}}{\left\|\mathbf{u}_{i}-\mathbf{u}_{j}\right\|} \mathbf{0}_{1 \times 3(j-2)} \frac{-\left(\mathbf{u}_{i}-\mathbf{u}_{j}\right)^{\mathrm{T}}}{\left\|\mathbf{u}_{i}-\mathbf{u}_{j}\right\|} \mathbf{0}_{1 \times 3(N-i-j+1)}\right], \quad i=1,2, \ldots, N-1 j=2,3, \ldots, N,
\end{aligned}
$$

where the $(\bullet)^{+}$denotes pseudo-inverse.

It can be seen that $\mathbf{Q}_{\mathbf{u}}$ has already contained the exact prior information of relative distance among the sources, which leads to a reduction in CRB. Hence, we have the following constrained CRB (CCRB) matrix of the lowest variance for the efficient constrained estimator $\widehat{\mathbf{u}}$ as

$$
\mathrm{C}_{\mathrm{CCRB}}=\mathbf{B}_{\mathbf{u}} \text {. }
$$

The diagonal elements of $\mathbf{C}_{C C R B}$ give the lowest estimate variance of the source coordinates. For example, in the $m$ dimensional scenario, the CCRB for $\mathbf{u}_{j}$ in this case is the sum of the $j^{\text {th }} m$ diagonal elements of $\mathbf{C}_{C C R B}$ after taking square root operation.

\section{Simulation Results}

In this section, simulation has been conducted to evaluate the TDOA-based localization performance of the proposed SDR algorithm. The localization algorithm developed in this paper for multiple sources, denoted as "SDP-CL", is compared with the single target SDR algorithm which is similar to [7] using only TDOA measurements, namely, the "SDP-SL", and the classic two-step weighted least-square (TS-WLS) method [4]. The CRB for single target case [5] and CCRB derived in this paper are also included.

In the simulation, six stationary sensors are employed to locate more than two targets in the sensor network. The positions of the sensors are listed in Table 2; they are the same as the setting in [1].

The positioning performance is evaluated by the root mean square errors (RMSE), defined by

$$
\operatorname{RMSE}=\left[\frac{\left(\sum_{i=1}^{L}\|\widehat{\mathbf{u}}-\mathbf{u}\|^{2}\right)}{L}\right]^{1 / 2},
$$

where $\widehat{\mathbf{u}}$ is the estimate of the true source position $\mathbf{u}$ and the RMSE of $j^{\text {th }}$ source position $\mathbf{u}_{j}(j=1,2 \ldots, N)$ can be directly computed in the same way when the estimate $\widehat{\mathbf{u}}_{j}$ is 


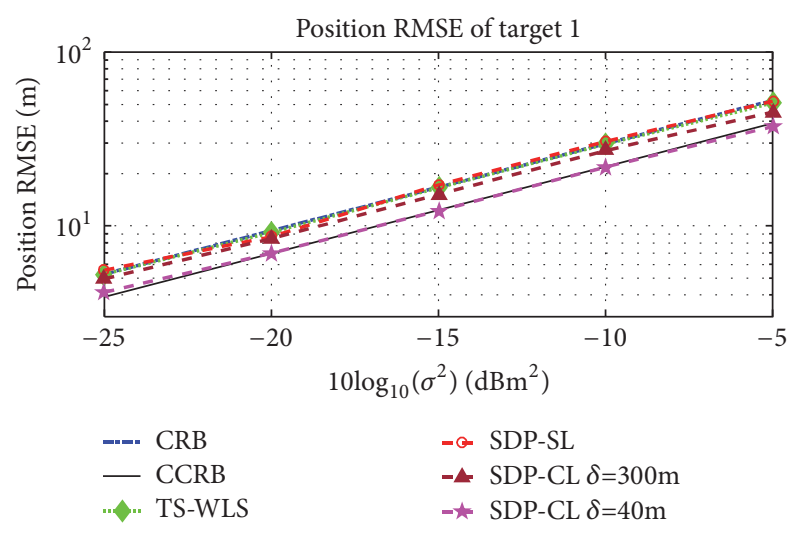

(a) RMSE of target 1

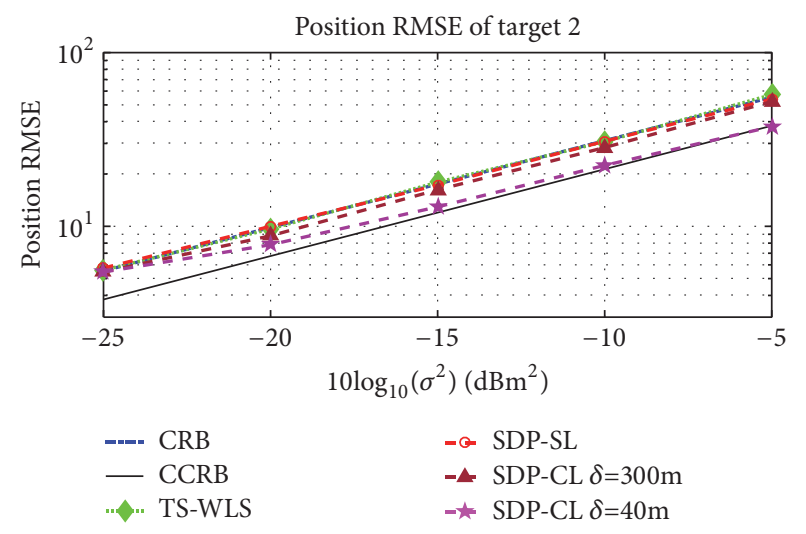

(b) RMSE of target 2

FIGURE 1: Comparison of RMSE and measurement noise $\sigma^{2}$ for two targets with different $\delta$.

obtained. The RMSE is obtained through $L=500$ independent Monte-Carlo runs in our simulation. The RDOA measurement noise is assumed to be a correlated Gaussian process [5], and its covariance matrix is of the form $\mathbf{Q}_{\Delta}=$ $\sigma^{2} \operatorname{blkdiag}\{\boldsymbol{\Sigma}, \boldsymbol{\Sigma}, \ldots, \boldsymbol{\Sigma}\}$ with

$$
\boldsymbol{\Sigma}=2 \times\left[\begin{array}{cccc}
1 & 0.5 & \cdots & 0.5 \\
0.5 & 1 & \cdots & 0.5 \\
\vdots & \vdots & \ddots & \vdots \\
0.5 & 0.5 & \cdots & 1
\end{array}\right]
$$

where $\boldsymbol{\Sigma}$ is the $(M-1) \times(M-1)$ matrix, and $\mathbf{Q}_{\Delta}$ is the $N(M-1) \times N(M-1)$ matrix. In simulation, the value of the range difference measurement error variance $\sigma^{2}$ is modified to achieve different noise conditions, and the measurement noises of different sources are assumed to be independent. The SDP in this paper is solved using the Matlab toolbox CVX [22], where the solver is SDPT3 [23].

In the first test, the impact of $\delta$ for localization performance is first investigated here. Without loss of generality, the group targets are assumed to consist of two emitting sources. The sources are located at 652,805 , and $710 \mathrm{~m}$ and 650,814 , and $708 \mathrm{~m}$. Thus the relative distance between them is about $10 \mathrm{~m}$, and we examine two situations where the upper range bound $\delta$ is set as $300 \mathrm{~m}$ and $40 \mathrm{~m}$, respectively. The estimated position RMSEs of the two targets against the measurement error variance $\sigma^{2}$ are shown in Figure 1. At $\delta=300 \mathrm{~m}$ and $40 \mathrm{~m}$, Figure 1 shows that when the upper range bound is more precise, the proposed method can achieve better result than that of large $\delta$ and eventually attain the corresponding CCRB. It can also be noticed that even when $\delta$ is large, for example, $\delta=300 \mathrm{~m}$, our method can slightly improve the localization accuracy compared with the localization algorithms for single source. Additionally, it can be observed from Figure 1 that the localization RMSE of the proposed method can approach the CCRB as the measurement error gradually grows large. This indicates that the range constraints between sources are more effective as the measurement noise increases. The result corresponds to the analysis of Remark 2 in Section 3.

In the second test, the number of targets is increased to three. Their positions are 654,803 , and $708 \mathrm{~m}, 652,808$, and $710 \mathrm{~m}$, and 650,814 , and $705 \mathrm{~m}$. Thus the true ranges between the targets are approximately up to $12 \mathrm{~m}$. Here the observed upper range bound information $\delta$ is set as $60 \mathrm{~m}$. The simulations results are plotted in Figure 2, from which we can see that the performance of proposed collaborative localization method outperforms other methods. And it can be also noticed that the localization accuracy can be improved with the aid of the range constraints, as the measurement error gradually becomes intensive. Moreover, based on the above two tests, it can be found that our method can yield a promotion of localization accuracy by utilizing a rough upper range bound when the range information between sources is not known precisely.

In the third test, the group targets are assumed to consist of three emitting sources that are farther from the sensors than that in the second test. Their positions are 1260, 1440, and $1193 \mathrm{~m}, 1256,1450$, and $1195 \mathrm{~m}$, and 1251, 1456, and $1188 \mathrm{~m}$. Thus the maximum relative distance among them is around $20 \mathrm{~m}$ and the upper range bound $\delta$ is set as $90 \mathrm{~m}$. The estimated position RMSEs of the three targets against the measurement error variance $\sigma^{2}$ are shown in Figure 3 when $\delta=90 \mathrm{~m}$. We can observe that the RMSE of the proposed position estimates using the SDP method is much lower than the results of traditional single target localization algorithms, and it can attain the corresponding $\mathrm{CCRB}$ as the measurement error increases. Comparing the results of the third test with those of the second test, the localization performance of all the three algorithms in the third test is worse than that in the second test because of the poor geometry. Nevertheless, our method can yield a promotion of localization accuracy by utilizing the range constraints compared with other algorithms in the both tests.

\section{Conclusion}

In this paper, we propose to jointly localize multiple emitting sources by utilizing the prior relative distance information 


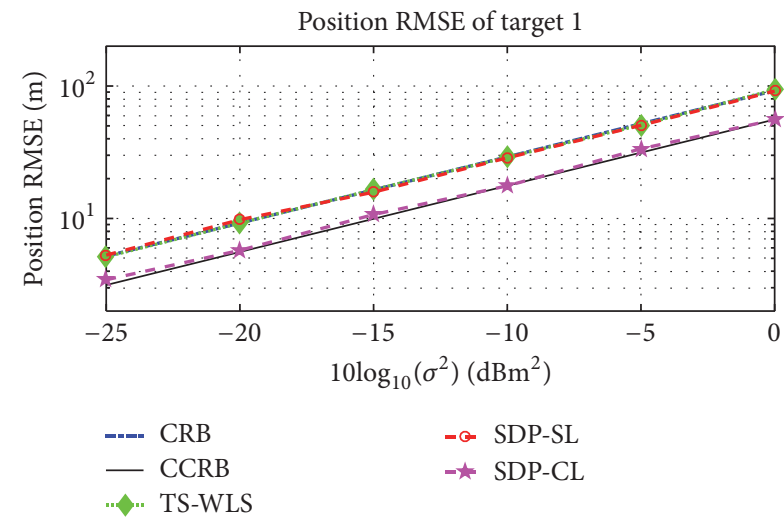

(a) RMSE of target 1

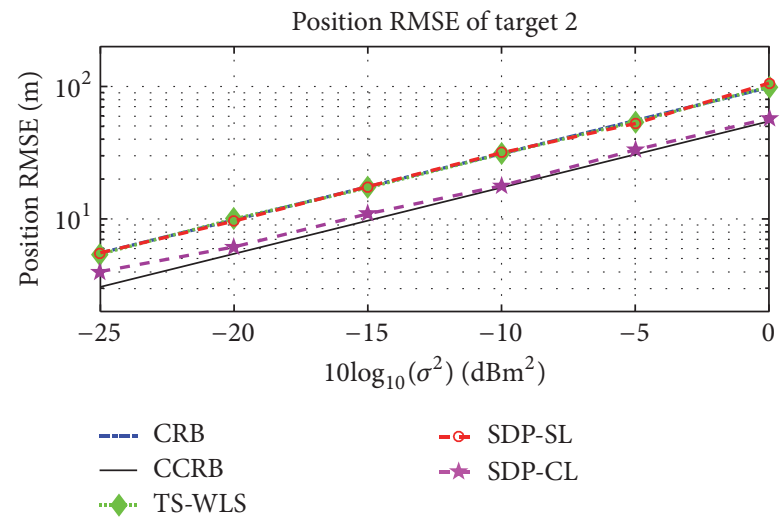

(b) RMSE of target 2

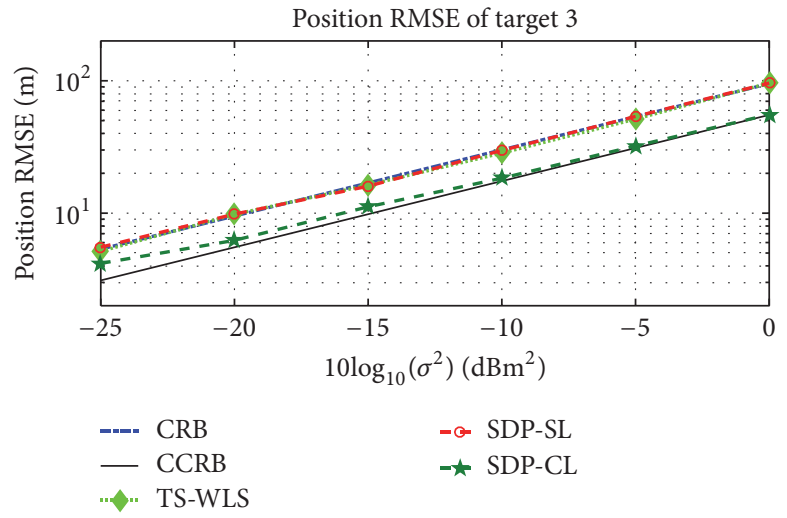

(c) RMSE of target 3

FIGURE 2: Comparison of RMSE and measurement noise $\sigma^{2}$ for three targets with $\delta=60 \mathrm{~m}$.

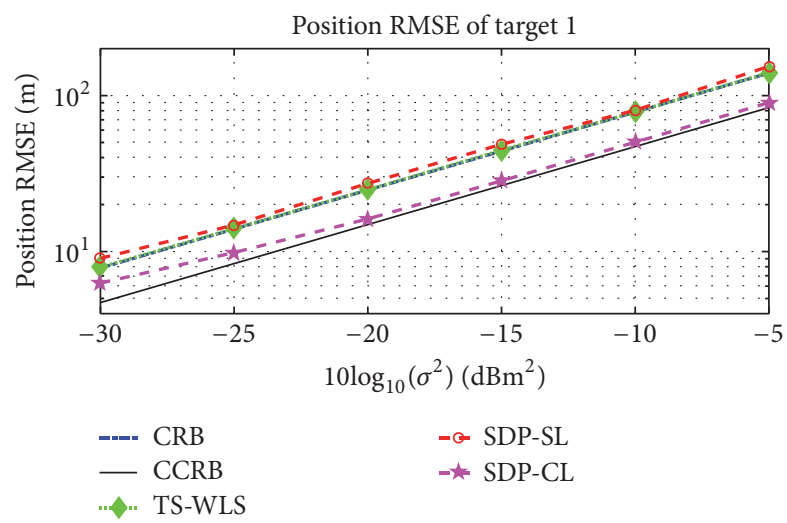

(a) RMSE of target 1

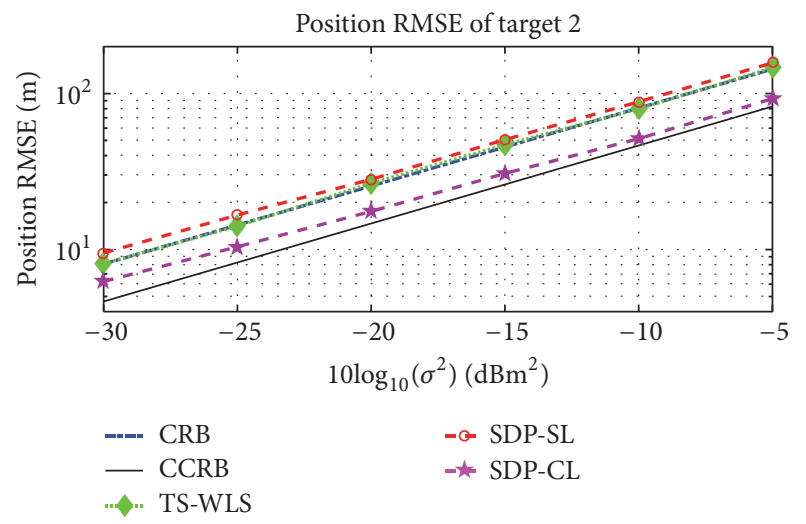

(b) RMSE of target 2

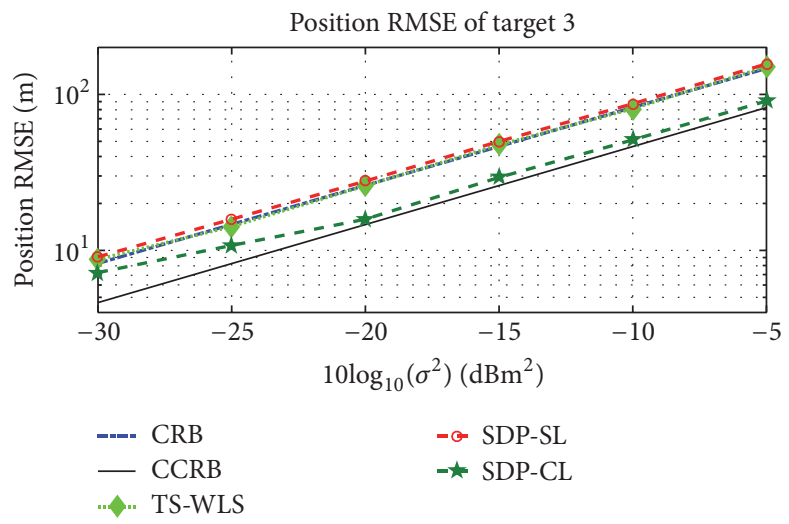

(c) RMSE of target 3

Figure 3: Comparison of RMSE and measurement noise $\sigma^{2}$ for three targets with $\delta=90 \mathrm{~m}$. 
between them based on TDOA measurements. The SDR technique is applied to reformulate the original nonconvex ML problem for multisource to obtain an SDP. Moreover, the constrained CRB, which incorporates the inequality constraints of prior relative distance information, is also derived in this paper. Simulation results show that the proposed method significantly improves the localization accuracy and can achieve the corresponding CCRB when the range information is more precise. In addition, the proposed method performs well even when the measurement error is intensive.

\section{Data Availability}

The authors claim that the data used in this article are provided by their simulations according to some real localization scenarios, and this article is developed without using any data in a published article to support their results.

\section{Conflicts of Interest}

The authors declare that they have no conflicts of interest.

\section{Acknowledgments}

The authors acknowledge support from the National Natural Science Foundation of China (Grants no. 61201381, no. 61401513, and no. 61772548), China Postdoctoral Science Foundation (Grant no. 2016M592989), the Self-Topic Foundation of Information Engineering University (Grant no. 2016600701), and the Outstanding Youth Foundation of Information Engineering University (Grant no. 2016603201).

\section{References}

[1] L. Yang and K. C. Ho, "An approximately efficient TDOA localization algorithm in closed-form for locating multiple disjoint sources with erroneous sensor positions," IEEE Transactions on Signal Processing, vol. 57, no. 12, pp. 4598-4615, 2009.

[2] D. Carevic, "Automatic estimation of multiple target positions and velocities using passive TDOA measurements of transients," IEEE Transactions on Signal Processing, vol. 55, no. 2, pp. 424-436, 2007.

[3] C. Mensing and S. Plass, "Positioning Algorithms for Cellular Networks Using TDOA," in Proceedings of the 2006 IEEE International Conference on Acoustics Speed and Signal Processing, vol. 4, pp. 513-516, Toulouse, France, 2006.

[4] K. C. Ho and W. Xu, "An accurate algebraic solution for moving source location using TDOA and FDOA measurements," IEEE Transactions on Signal Processing, vol. 52, no. 9, pp. 2453-2463, 2004.

[5] K. W. K. Lui, F. K. W. Chan, and H. C. So, "Semidefinite programming approach for range-difference based source localization," IEEE Transactions on Signal Processing, vol. 57, no. 4, pp. 1630-1633, 2009.

[6] E. Xu, Z. Ding, and S. Dasgupta, "Reduced complexity semidefinite relaxation algorithms for source localization based on time difference of arrival," IEEE Transactions on Mobile Computing, vol. 10, no. 9, pp. 1276-1282, 2011.

[7] Y. Wang and Y. Wu, "An efficient semidefinite relaxation algorithm for moving source localization using TDOA and
FDOA measurements," IEEE Communications Letters, vol. 21, no. 1, pp. 80-83, 2017.

[8] G. Wang, Y. Li, and N. Ansari, "A semidefinite relaxation method for source localization using TDOA and FDOA measurements," IEEE Transactions on Vehicular Technology, vol. 62, no. 2, pp. 853-862, 2013.

[9] F. Giulietti, L. Pollini, and M. Innocenti, "Automatic formation flight," IEEE Control Systems Magazine, vol. 20, pp. 34-44, 2000.

[10] M. Hernandez, "Novel maximum likelihood approach for passive detection and localisation of multiple emitters," EURASIP Journal on Advances in Signal Processing, vol. 2017, p. 36, 2017.

[11] Y. Lee, T. S. Wada, and B.-H. Juang, "Multiple acoustic source localization based on multiple hypotheses testing using particle approach," in Proceedings of the 2010 IEEE International Conference on Acoustics, Speech, and Signal Processing, ICASSP 2010, pp. 2722-2725, Dallas, Tex, USA, March 2010.

[12] S. Venkateswaran and U. Madhow, "Localizing multiple events using times of arrival: a parallelized, hierarchical approach to the association problem," IEEE Transactions on Signal Processing, vol. 60, no. 10, pp. 5464-5477, 2012.

[13] H. Shen, Z. Ding, S. Dasgupta, and C. Zhao, "Multiple source localization in wireless sensor networks based on time of arrival measurement," IEEE Transactions on Signal Processing, vol. 62, no. 8, pp. 1938-1949, 2014.

[14] J. Li, H. Pang, F. Guo, L. Yang, and W. Jiang, "Localization of multiple disjoint sources with prior knowledge on source locations in the presence of sensor location errors," Digital Signal Processing, vol. 40, pp. 181-197, 2015.

[15] L. Doherty, K. S. J. Pister, and L. E. Ghaoui, "Convex position estimation in wireless sensor networks," IEEE INFOCOM, vol. 3, pp. 1655-1663, 2001.

[16] K. W. Cheung, W.-K. Ma, and H. C. So, "Accurate approximation algorithm for TOA-based maximum likelihood mobile location using semidefinite programming," in Proceedings of the 2004 IEEE International Conference on Acoustics, Speech, and Signal Processing, vol. 2, pp. 145-148, Montreal, Canada, 2004.

[17] S. Boyd and L. Vandenberghe, Convex Optimization, Cambridge University Press, Cambridge, UK, 2004.

[18] Y. T. Chan and K. C. Ho, "A simple and efficient estimator for hyperbolic location," IEEE Transactions on Signal Processing, vol. 42, no. 8, pp. 1905-1915, 1994.

[19] T. Strutz, Data Fitting and Uncertainty: A Practical Introduction to Weighted Least Squares and Beyond, Vieweg and Teubner, 2nd edition, 2016.

[20] L. Vandenberghe and S. Boyd, "Semidefinite programming," SIAM Review. A Publication of the Society for Industrial and Applied Mathematics, vol. 38, no. 1, pp. 49-95, 1996.

[21] J. D. Gorman and A. O. Hero, "Lower bounds for parametric estimation with constraints," Institute of Electrical and Electronics Engineers Transactions on Information Theory, vol. 36, no. 6, pp. 1285-1301, 1990.

[22] M. Grant and S. Boyd, CVX: Matlab Software for Disciplined Convex Programming, 2009, Available: http://stanford.edu/ boyd/cvx.

[23] K. C. Toh, M. J. Todd, and R. H. Tutuncu, "SDPT3-a MATLAB software package for semidefinite programming, version 1.3," Optimization Methods and Software, vol. 11, no. 1, pp. 545-581, 1999. 


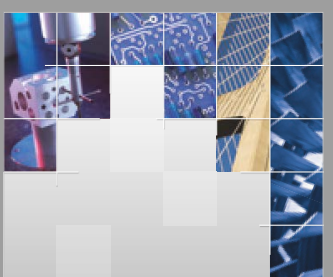

\section{Enfincering}
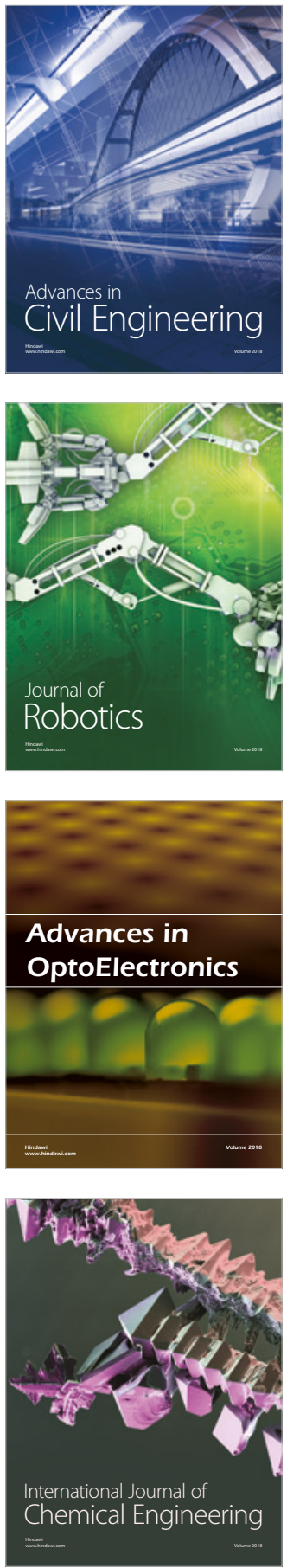

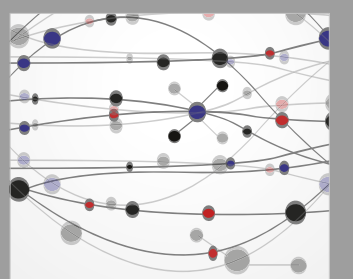

\section{Rotating \\ Machinery}

The Scientific World Journal

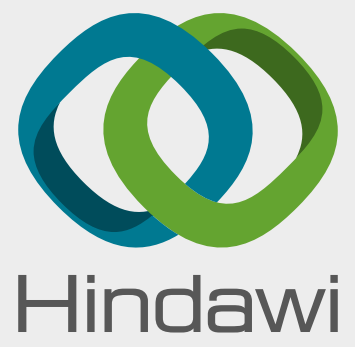

Submit your manuscripts at

www.hindawi.com
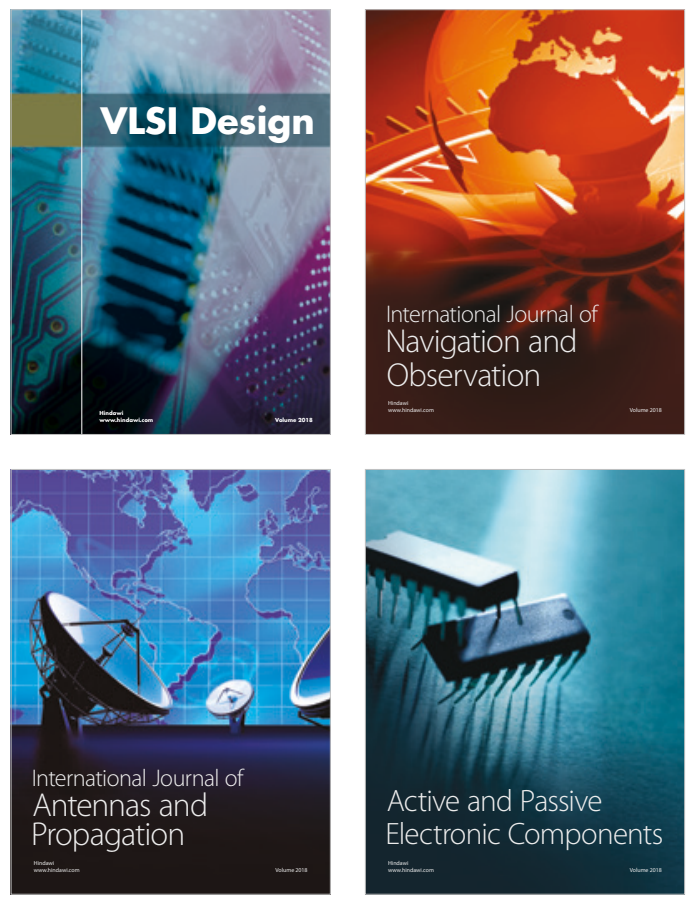
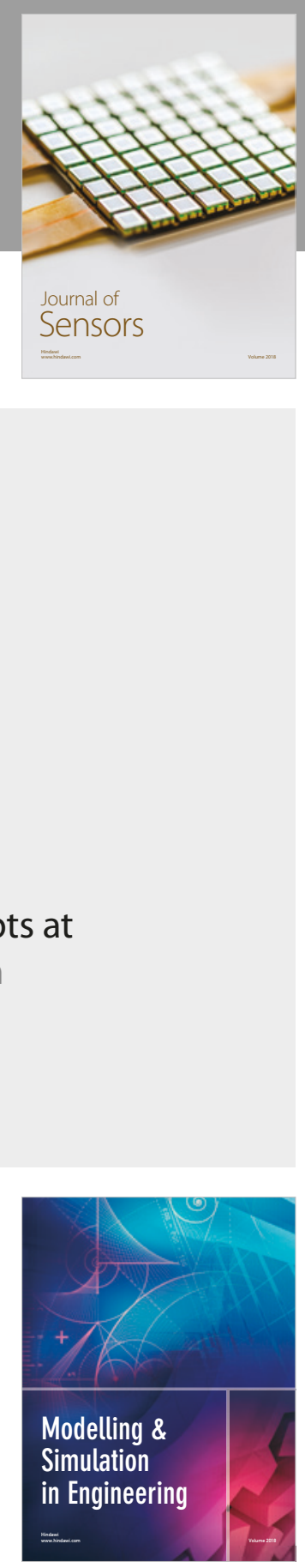

\section{Advances \\ Multimedia}
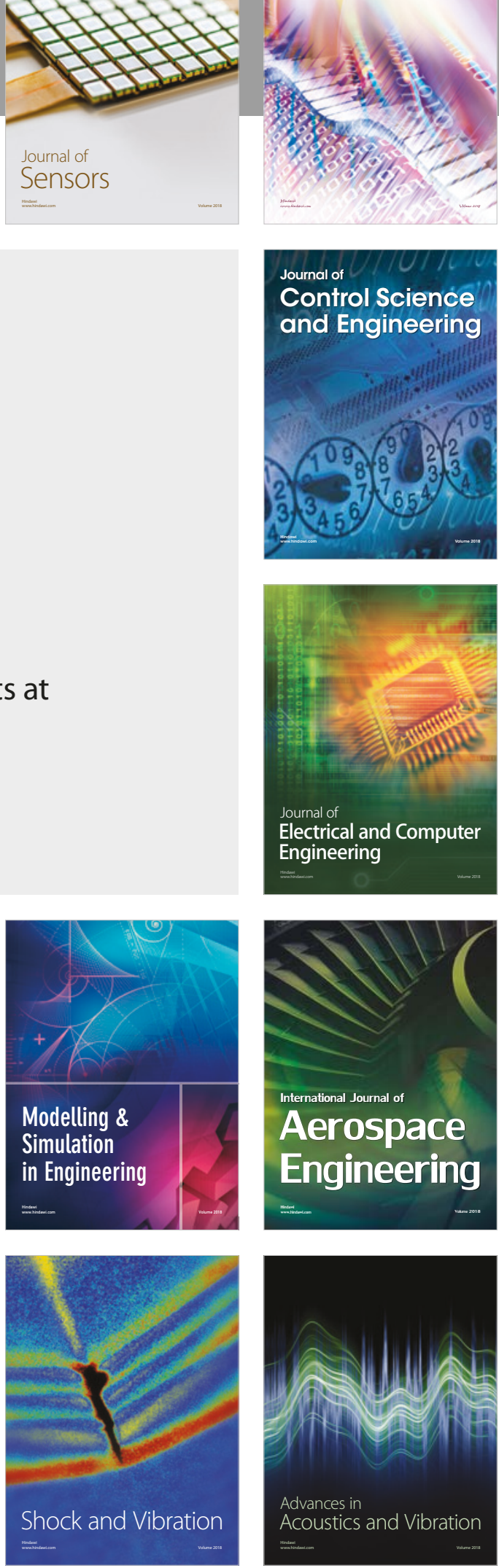